\title{
The role of the alloy structure in the magnetic behavior of granular systems
}

\author{
C. S. M. Bastos, M. Bahiana, W. C. Nunes and M. A. Novak \\ Instituto de Física, Universidade Federal do Rio de Janeiro \\ Caixa Postal 68528, Rio de Janeiro, RJ, Brazil, 21945-970 \\ D. Altbir \\ Departamento de Física, Universidad de Santiago de Chile \\ Casilla 30\%, Santiago 2, Chile \\ P. Vargas \\ Departamento de Física, \\ Universidad Técnica Federico Santa María \\ Casilla 110-V, Valparaíso, Chile \\ M. Knobel \\ Instituto de Física "Gleb Wataghin", Universidade Estadual de Campinas (UNICAMP) \\ CP 6165, 13083-970, Campinas, São Paulo, Brazil
}

(Dated: November 6, 2018)

\begin{abstract}
The effect of grain size, easy magnetization axis and anisotropy constant distributions in the irreversible magnetic behavior of granular alloys is considered. A simulated granular alloy is used to provide a realistic grain structure for the Monte Carlo simulation of the ZFC-FC curves. The effect of annealing and external field is also studied. The simulation curves are in good agreement with the $\mathrm{FC}$ and $\mathrm{ZFC}$ magnetization curves measured on melt spun $\mathrm{Cu}-\mathrm{Co}$ ribbons.
\end{abstract}

\section{INTRODUCTION}

Besides the importance of studying still open problems on basic magnetism, nanocrystalline systems attract more and more interest due to their applications on chemical catalysis and magnetic recordingt. For the latter, the progressive increase of recording density has lead to the production of materials with smaller single domain particles. This requirement has the serious drawback that the effective magnetic moment of the particles suffer strong instabilities of thermal origin, so called superparamagnetic limit. Also, when one deals with the nanometer scale, the magnetic systems are not easily reproduced and characterized, introducing additional difficulties for experimental studies. Experimental and theoretical results over the past years show that there are clearly many factors that can influence the magnetic and magnetotransport behavior of these systems, namely: The distribution of grain sizes, the average size and shape of the grains, the magnetic anisotropy of the individual grains and magnetic interactions among the nanometer-sized crystallites.

The effect of some of these parameters on the magnetization and magnetoresistance has been partially investigated by several authors. El-Hilo et al 1 have used Monte Carlo simulations for determining the magnetoresistance dependence on the mean intergranular distance, or rather, the particle concentration, using a simple expression previously obtained by Gittleman et al.3. The influence of the log-normal distribution of magnetic moments and the ratio of the boundary to the volume scattering cross sections on the magnetization and magnetoresistance has been examined by Ferrari et al. . Hickey et al. 5 and Wisen have developed a phenomenological model which explains the almost linear variation of the magnetoresistance with the magnetization at low temperatures as a possible consequence of existing correlations between blocked and superparamagnetic particles. The blockine effect on the magnetization curves has been simulated by a Monte Carlo method in a paper of Dimitrov and Wysint. Allia et al 8 have proposed analytical models that take explicitly into account the correlation arising from the dipolar interactions on nearly superparamagnetic systems. Also for interacting systems, Chantrell et al. 8 calculated the susceptibillity and Field-Cooling (FC) and Zero Field-Cooled (ZFC) curves for superparamagnetic particles, and Pike et al. 10 investigated the role of magnetic interactions on low temperature saturation remanence of fine magnetic particles. However, most of these models assume non-realistic size distributions of the grains, and constant anisotropy, as if all grains had the same shape. Both parameters originate effects that certainly are interesting to elucidate.

In order to investigate the effect of different structural and magnetic factors on the magnetization properties of granular alloys we have simulated, by means of a standard Monte Carlo method, one of the most common characterization techniques, the FC-ZFC curves. The samples were simulated by a Cell Dynamical System model which gives the realistic grain sizes and shapes distributions and, with the aid of experimental results, these quantities can be assigned to magnetization and anisotropy for each grain. After these quantities are known, the magnetic properties 
of the system are studied as a function of temperature, applied field and thermal treatment of the sample.

Comparisons between the realistic distribution and others are made to check the influence of grain sizes and shape distributions on ZFC-FC curves. An interesting correspondence is made between annealing and time iteration in CDS. The applied field effects on blocking temperatures and magnetization values for ZFC-FC curves are studied and agree with experiments. Our results are compared with experimental results also included is this work.

\section{SIMULATION OF THE GRANULAR ALLOY}

Granular materials corresmond to metastable states resulting from the slowing down of the segregation process in binary off-critical mixtures1. In the case of metal alloys, a quench to the metastable region of the phase diagram generates a virtually permanent granular state at room temperature. Since the grain structure is not an equilibrium state, it cannot be derived from a minimization procedure. There are several statistically equivalent granular structures corresponding to different paths in phase space and one must go through the process of phase separation in order to reach one of these intermediate states and obtain realistic simulations of granular alloys.

The traditional approach to this problem is the Cahn-Hilliard equation 12

$$
\frac{\partial \psi(\mathbf{r}, t)}{\partial t}=L \nabla^{2} \frac{\delta F[\psi(\mathbf{r}, t)]}{\delta \psi(\mathbf{r}, t)}
$$

where $\psi(\mathbf{r}, t)$ is a conserved order parameter (in our case, the difference between Co and $\mathrm{Cu}$ concentrations), $L$ is a phenomenological parameter related to the mobility, and $F[\psi(\mathbf{r}, t)]$ is the coarse-grained Landau free-energy functional:

$$
F[\psi(\mathbf{r}, t)]=\int d \mathbf{r}\left[\frac{1}{2}(\nabla \psi)^{2}-\frac{\tau}{2} \psi^{2}+\frac{g}{4} \psi^{4}\right],
$$

with $\tau$ and $g$ positive phenomenological parameters. In principle, the solution of equation (1) yields the knowledge of the whole phase separation process. The problem is, of course, that (11) does not have an analytical solution and it is very hard to solve numerically. Almost all efforts in this sense were able to describe the very early stages of the process only. Other common approachesare Aonte farlo simulations with Kawasaki exchange dynamics 13.14 and Cell

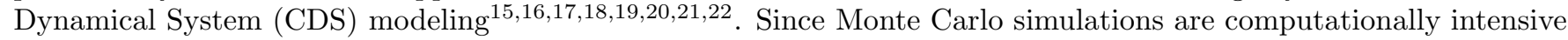
and CDS has been successfully used to model this type of problems, we have chosen the latter.

The basic point of CDS modeling is the discreteness of space and time. The point of view is equivalent to the one in the Cahn-Hilliard equation, in the sense that a coarse grained description based on densities is used, and the parameters are phenomenological. The stability of the dynamics and the computational efficiency allows the achievement of the asymptotic regime even for reasonably large systems. The disadvantage of this approach is that the arbitrary definition of parameters does not yield absolute information about the system, although this can be done in some cases by recovering the correct time and space scales22.

The simulation, in this case, mimics an experiment in which a binary alloy is initially prepared at a temperature above the phase separation critical temperature, with given amounts of each component, and then quenched to a region of the phase diagram in which the homogeneous phase is metastable. The system is represented by a $d$-dimensional lattice and its configurations at time $t$ and $t+1$ are directly related by a map. Here we have considered only $d=2$, since we believe that most of the properties we seek will be manifested in this dimension, so the computational effort involved in a $3 d$ simulation would not improve significantly our results.

We assume that the dynamics of each lattice cell is governed by a local relaxational mechanism driven by a suitable map $f$. The exact form of the map is not important, as long as it has the correct flow 15.26 .23 . For the segregation of binary mixtures we seek a map with one unstable fixed point at the origin and two hyperbolically stable fixed points at symmetrical positions. The stable fixed points correspond to the segregated phases rich in $\mathrm{Co}$ and $\mathrm{Cu}$, and the unstable fixed point, to the homogeneous phase.

The single cell dynamics is described by

$$
\psi(t+1, n)=f[\psi(t, n)],
$$

where $\psi(t, n)$ is the value of the order parameter in the cell $n$ at time $t$. The addition of a diffusional coupling to its neighborhood leads to a non conservative dynamics of the form

$$
\begin{aligned}
\psi(t+1, n) & =f[\psi(t, n)]+D[\langle\langle\psi(t, n)\rangle\rangle-\psi(t, n)] \\
& =\psi(t, n)+I(t, n)
\end{aligned}
$$


where $I(t, n)=f[\psi(t, n)]-\psi(n, t)+D[\langle\langle\psi(n, t)\rangle\rangle-\psi(n, t)]$ is the increment in order parameter after one iteration, and $D$ is a positive parameter proportional to the phenomenological diffusion constant. $\langle\langle *\rangle\rangle-*$ is the isotropic discrete Laplacian. We use the following definition of spatial average $\langle\langle *\rangle\rangle$ on the two-dimensional square lattice:

$$
\langle\langle\psi(t, n)\rangle\rangle=\frac{1}{6} \sum_{n n} \psi+\frac{1}{12} \sum_{n n n} \psi,
$$

where the sums are over nearest-neighbors and next-nearest-neighbors cells respectively. The conservation of order parameter may be locally imposed by the subtraction of the average gain in order parameter in the neighborhood of each cell. The conserved order parameter CDS model then reads

$$
\psi(t+1, n)=\psi(t, n)+I(t, n)-\langle\langle I(t, n)\rangle\rangle .
$$

\section{A. Simulations conditions}

We used a two-dimensional lattice with $450^{2}$ cells and periodic boundary conditions. The order parameter represents the difference between the average concentration of $\mathrm{Cu}$ and $\mathrm{Co}$ in the alloy, $\psi=\psi_{\mathrm{Cu}}-\psi_{\mathrm{Co}}$. Our initial condition is the homogeneous system with $\psi(\vec{r})=\psi_{0}+\delta(\vec{r})$, where $\delta(\vec{r})$ is a small random fluctuation uniformly distributed in the interval $[-0.005,0.005]$. For a $\mathrm{Cu}_{90} \mathrm{Co}_{10}$ alloy, $\left\langle\psi_{\mathrm{Cu}}\right\rangle=0.9$ and $\left\langle\psi_{\mathrm{Co}}\right\rangle=0.1$ (average values of concentration), so we have $\psi_{0}=0.8$. For such values of concentration the system is in a metastable state after the quench, so phase ordering proceeds via nucleation, and segregation of phases can occur only if nucleation centers are present. In our simulation nucleation centers consisting of four sites each with $\psi=-0.5$, adding up to apout $10 \%$ of the lattice were randomly placed. For a longer discussion about off-critical quench in CDS models see 6 . We used $D=0.5$ and our choice of map is

$$
f(x)=\mathcal{A} \tanh x
$$

where $\mathcal{A}$ is a measure of the quench depth $(\mathcal{A}>1$ corresponds to a homogeneous mixture). In our simulations we use $\mathcal{A}=1.3$. Figure 1 shows the snapshots of the lattice after 100, 1000, 5000, 10000 iterations. The color black is associated to the presence of Co, so, according to our definition of $\psi$, the grains have $\psi<0$. The time evolution of the pattern is driven by the gradient of the chemical potential, leading to domain coarsening and coalescence, so that patterns obtained after different number of iterations have distinct distributions of grain sizes and shapes, leading to distinct magnetic properties. As the segregation process proceeds, grains coalesce and grow, such that the number of grains decrease, as they become larger. In real samples such evolution of the grain structure is possible only with a thermal treatment. In this way, we are going to compare patterns simulated at later times with experimental samples that have been annealed at higher temperatures.

\section{B. Analysis of the grain structure}

We have used the standard algorithm developed by Hoshen and Kopelman24 to obtain the list of cells belonging to each grain. In order to check the form of the grain size distribution we have calculated the histograms corresponding to granular patterns after given number of iterations of Eq. 7 in terms of the magnetic moments $m$ of the grains. As explained below, the magnetic moments are proportional to the number of sites in each cluster with $\psi<0$. Figure 2 shows the histograms after 1000 (Sample 1), and 10000 (Sample 2) iterations, showing that as time goes on there is a shift to larger values of magnetization, due to the coalescence of grains. In order to compare the distribution obtained in simulated samples to the usually adopted log-normal distribution, we have fitted the above histograms with the curve

$$
P(m) \mathrm{d} m=\frac{A}{(\sqrt{2 \pi}) w m} \exp \left[-\frac{\left(\ln \frac{m}{m_{c}}\right)^{2}}{2 w^{2}}\right] \mathrm{d} m,
$$

where $P(m)$ is the probability density to find the grain magnetic moment between $m$ and $m+\mathrm{d} m, m_{c}$ is the most probable value of $m$, and $w$ is the width of the distribution.

The resulting fitting parameters are summarized in Table [ 


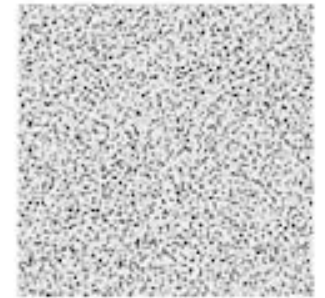

(a)

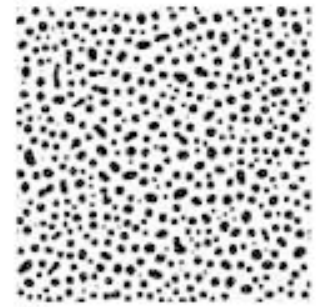

(c)

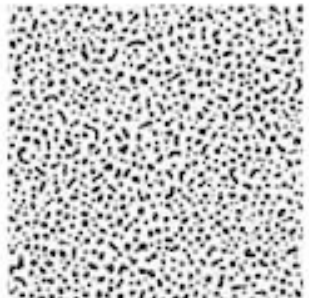

(b)

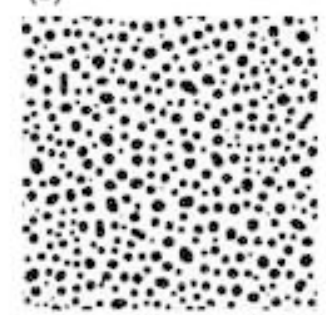

(d)

FIG. 1: Segregation patterns for a $\mathrm{Cu}_{90} \mathrm{Co}_{10}$ alloy after (a) 100, (b) 1000, (c) 5000, and (d) 10000 iterations of Eq. (8). Darker shades of gray correspond to Co rich regions.
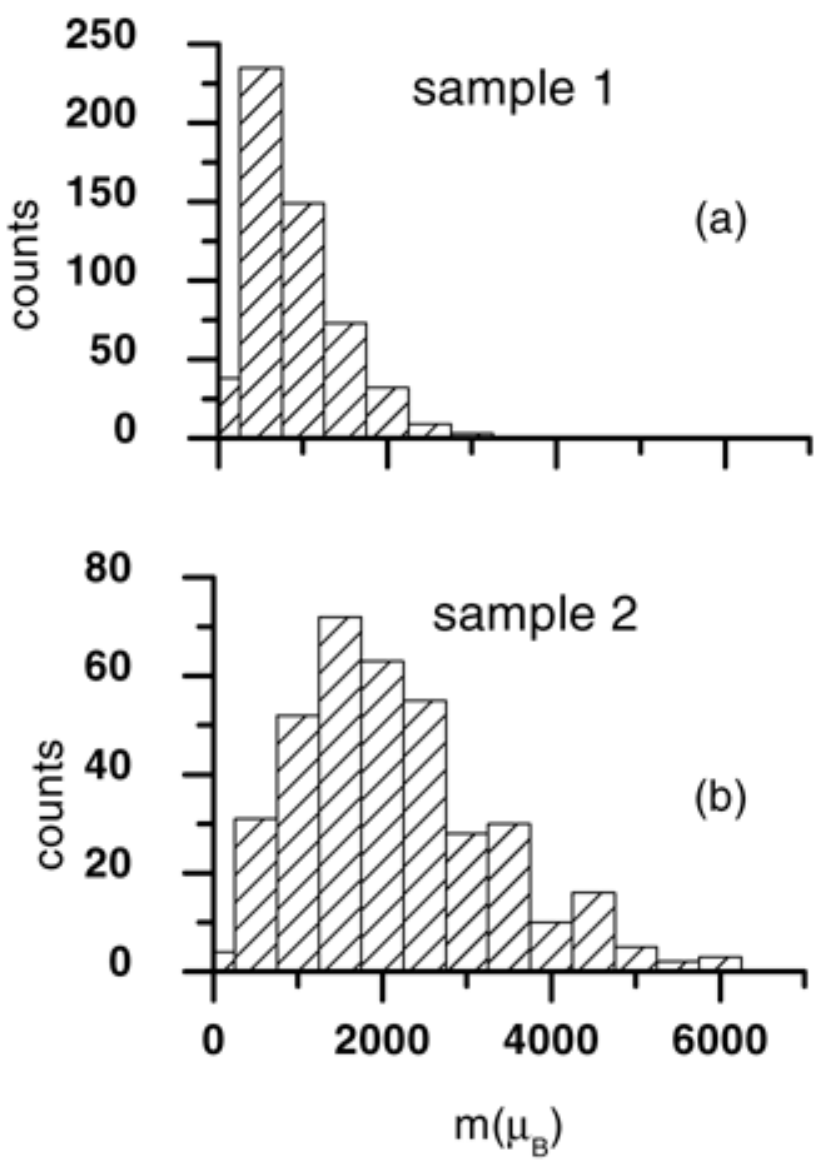

FIG. 2: Histograms for the patterns after 1000, (a), and 10000, (b), iterations shown in Fig. 11. 


\begin{tabular}{|c|c|c|c|}
\hline sample & $m_{c}\left(\mu_{B}\right)$ & $w$ & $A\left(\mu_{B}\right)$ \\
\hline \hline 1 & 823 & 0.80 & $116 \times 10^{3}$ \\
\hline 2 & 2091 & 0.63 & $189 \times 10^{3}$ \\
\hline
\end{tabular}

TABLE I: Parameters resulting from the fitting of a Log-normal distribution defined by Equation (9) to the histograms of Fig. 2.

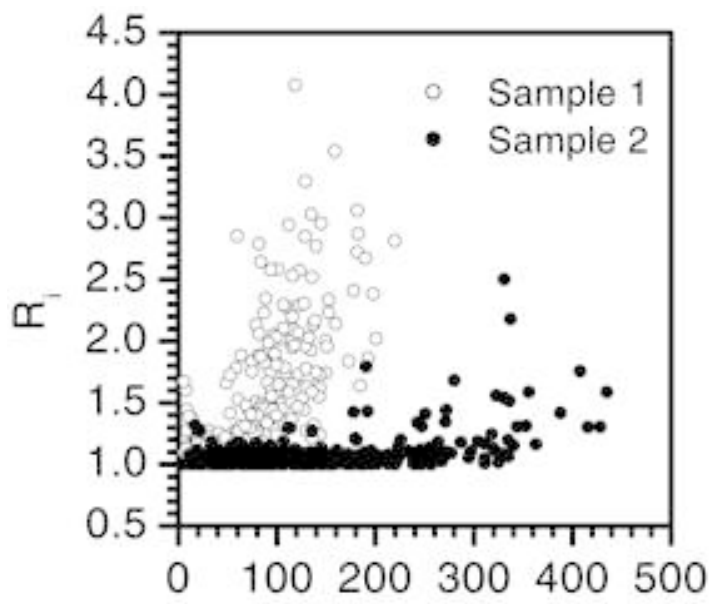

$n_{1}$

FIG. 3: Scatter plot of the anisotropy, measured as the ration $R_{i}$ between the larger and the smaller dimension of the $i$-th grain, as a function of the number of sites, $n_{i}$ in the grain. It is clear from this plot that the segregation process leads to larger and rounder grains, and that anisotropy is correlated to grain size. We expect the same behavior in real systems, that is, annealing will generate a system with larger but less anisotropic grains.

Cobalt grains have fcc symmetry 25 , so it is reasonable to consider that the dominant contribution to the anisotropy energy comes from the grains shape. Observing the pattern formed after 1000 iterations (Fig. 1. (b)), for example, we notice essentially two kinds of grain shapes, one almost circular, and another elongated. Once the grains are labeled we can obtain information about their shape in order to calculate the direction of the easy magnetization axis. The method for calculating this direction is quite simple, it consists in calculating the position of the central site of the $i$-th grain, and then summing up the position vectors of each site related to the position of this central site. The resultant vector direction is the easy axis direction, $\widehat{e}_{i}$.

Finally we have translated our arbitrary units to physical ones, using a real $\mathrm{Cub}_{90} \mathrm{Co}_{10}$ sample for which the average values of the grain magnetic moment and anisotropy constants are known. The determination of those experimental values is explained in Section IIC. The first step was to associate a magnetization value to each grain. Assuming that each grain magnetization is proportional to its size, we can adjust our results by comparing them to experimental data. Of course one serious difficulty is that the whole simulation is $2 \mathrm{~d}$, so actually we have areas and not volumes for the grains.

The average grain size is just the average number of lattice sites of the grains, $\bar{n}$, and was associated to the average experimental magnetic moment $m_{\exp }$ of a $\mathrm{Cub}_{90} \mathrm{Co}_{10}$ sample as

$$
\bar{n} f_{m}=m_{\text {expo }}
$$

so that the magnetic moment of the $i$-th simulated grain is then $m_{i}=n_{i} f_{m}$, where $n_{i}$ is the number of sites of that grain, and $f_{m}$ the conversion factor in units of $m_{\text {exp }}$.

We also need the conversion factor for the anisotropy constant $K$. We used the fact that, for cobalt, when $R_{i}=3.5$ the shape anisotropy constant is equal to $K_{\text {exp }}=4.5 \times 10^{6} \mathrm{erg} / \mathrm{cam}^{3} 26$. With this it is possible to define the conversion factor $f_{K}$ as

$$
f_{K}=\frac{K_{\text {expo }}}{3.5}
$$

so that, for each grain, the anisotropy constant in the same units of $K_{\text {exp }}$ may be calculated as $K_{i}=f_{K} R_{i}$.

The properties of the samples used in the Monte Carlo simulations are summarized in Table II. 


\begin{tabular}{|c|c|c|c|c|}
\hline sample & number of iterations & $N_{g}$ & $\bar{m}\left(\mu_{B}\right)$ & $\bar{K}\left(10^{6} \mathrm{erg} / \mathrm{cam}^{3}\right)$ \\
\hline \hline 1 & 1000 & 539 & 869 & 1.58 \\
\hline 2 & 10000 & 371 & 2140 & 1.58 \\
\hline
\end{tabular}

TABLE II: Properties of the simulated Cub90 $\mathrm{Co}_{10}$ samples used in the Monte Carlo simulations. $N_{g}$ is the total number of grains in a sample, $\bar{m}\left(\mu_{B}\right)$ is the average magnetic moment of the grains and $\bar{K}$ is the average anisotropy constant of the sample.

\section{Experiment}

Continuous ribbons of $\mathrm{Co}_{10} \mathrm{Cub}_{90}$ were obtained by melt spinning in $\mathrm{AR}$ atmosphere using a Cub-AR drum. The as cast material was subjected to furnace annealing at temperature range $400-600{ }^{\circ} \mathrm{C}$ for $60 \mathrm{~min}$, generating samples with different monostructures. Details of the sample structure and how it is affected by the thermal treatment can be seen in reference 27. The magnetization measurements were performed on a commercial Quantum Design PPMS system with temperatures in the range 2-300 K, for different values of the applied field $H$. In each curve the sample was cooled to $2 \mathrm{~K}$ in zero field. After applying a magnetic field, the magnetization was measured with increasing temperature up to $300 \mathrm{~K}$ (ZFC magnetization). Subsequently, the magnetization was measured for decreasing temperature in the same field down to $2 \mathrm{~K}$ (FC magnetization). The magnetic field was applied by a superconducting magnet operating in persistent mode and the total time of each complete ZFC and FC curves was of 7 hours with a rate of temperature sweep of $1.5 \mathrm{~K} / \mathrm{min}$.

The moment distribution of the samples was obtained by fitting a sym of Langevin functions weighted by a lognormal moment distribution in the $m(H)$ curve measured at $T=300 \mathrm{~K}$. The saturation magnetization was obtained by extrapolation of the curve $m(1 / H)$ to zero temperature.

\section{MONTE CARLO SIMULATION}

A ferromagnetic particle becomes a monodomain when its linear size is below a critical value $P_{\text {determined by the }}$ minimization of the total energy, including magnetostatic, exchange and anisotropy contributions 28 . Below this critical size, the energy associated to the creation of magnetic domain walls is larger than the decrease in the magnetostatic energy due to the smaller total magnetization. Such monodomain ferromagnetic particles can be viewed as large magnetic units, each having a magnetic moment of thousands of Bohr magnetons. Usually, in low concentration alloys, neighboring particles are separated by $10-30 \mathrm{~nm}$, and direct exchange, as well as indirect (like RKKY) between particles is neglected 2 . Thus, the magnetic properties of an assembly of nanoparticles are determined by the dipolar interaction energy between the particles along with thermal and magnetic anisotropy energies. In this paper we consider only the latter effect. Experiments conducted on magnetic nanoparticles show irreversible magnetic behavior below a irreversibility line $T_{i r r}(H)$. In particular, the ZFC and FC magnetization curves do not coincide, and magnetic hysteresis appears. In such systems the origin of irreversibility is the interplay between thermal energy and some energy barrier, which hinders relaxation towards equilibrium. The magnetic irreversibility in nanoparticles is conventionally associated with the energy required for a particle moment reorientation, overcoming a barrier due to shape, magnetoelasticity, or crystalline anisotropy 28 .

Our system consists of $N_{g}$ magnetic monodomain particles, whose sizes, shapes and anisotropy constants are obtained by means of the previously described procedure. Each particle is described by its magnetic moment $\vec{m}_{i}$, the direction of the easy magnetization axis, $\widehat{e}_{i}$ and its anisotropy constant $K_{i}$. All those quantities result from the CDS simulation of the granular structure. In the presence of an external magnetic field $\vec{H}$, the Hamiltonian of our system can be written as

$$
\mathcal{H}=\sum_{i}\left[-\vec{m}_{i} \cdot \vec{H}-\kappa_{i}\left(\frac{\vec{m}_{i} \cdot \widehat{e}_{i}}{m_{i}}\right)^{2}\right],
$$

where $\kappa_{i}=K_{i} N_{i}, N_{i}$ being the number of atoms of grain $i$.

The ZFC-FC curves correspond to nonequilibrium states of the system, therefore they are dependent on the temperature variation rate. In terms of a Monte Carlo simulation this means that we cannot wait too long at each value of temperature, and the usual mechanism of time averaging instead of ensemble averaging is not valid. The 
estimation of the number of Monte Carlo steps in each temperature was completely arbitrary and it has no obvious relation to the actual time used in experiments. However, as we keep this number constant in our simulations we can at least guarantee that the cooling and heating rates are equal in all simulations and our conclusions will be valid at these rates. The averaging was done over 200 independent but statistically equivalent sweeps. For the ZFC curve we started from a configuration where the magnetic moments of the grains were randomly chosen and $H=0$. The simulation proceeded by turning on the external field (typically 10-100 Oe) in the $x$-direction at low temperature $(T=2 \mathrm{~K})$. The $N_{g}$ grains were then sequentially chosen, and had their magnetic moment rotated by an angle sorted from a uniform distribution. The change in energy $(\Delta E)$ was calculated and the rotation accepted with probability $p=\min \left[1, \exp \left(-\Delta E / k_{B} T\right)\right]$. The update of the $N_{g}$ particles was repeated 100 times for the initial value of temperature, and then the temperature was increased by $2 \mathrm{~K}$, and so on. At $T=200 \mathrm{~K}$ we kept the last configuration of magnetic moments and used it as the initial configuration for the FC curve, following the same procedure used in the ZFC curve, only decreasing the temperature $2 \mathrm{~K}$ each step.

\section{RESULTS}

The analysis of ZFC curves usually involves two temperatures $T_{M}$ and $T_{i r r}$ defined as the temperature at the maximum and the temperature above which the system shows thermodynamic equilibrium properties corresponding to a superparamagnetic behavior, respectively 30 . For $T<T_{i r r}$ irreversibility impedes the coincidence of the ZFC and FC curves. For $T>T_{i r r}$, the relaxation time for the magnetization of the largest particle is much smaller than the typical measuring time, and the ZFC and FC curves coincide. Our goal is to understand how these temperatures are affected by the distribution of sizes and shapes of the particles, magnitude of the applied field and annealing of the grains.

\section{A. Influence of the distribution of grain sizes and shapes}

In order to compare the effect of the distribution of grain sizes and anisotropies on the ZFC-FC curves, we performed a series of simulations using different types of distributions namely, uniform, Gaussian and realistic (obtained from CDS simulation) for the sizes, anisotropy axis sorted from a uniform distribution of directions, and obtained from the CDS simulation, anisotropy constants equal to the average value, and calculated from the simulated alloy. One important point in the choice of the anisotropy constants $K_{i}$, is that they are intensive quantities. In order to keep the total anisotropy energy independent of the choice of the $K$ distribution, we calculated the average value of the anisotropy constant using the grain sizes as weights. The results below show the combination of those possibilities for the same sample under an applied external field $H=0.1 \mathrm{KOe}$. All curves show the behavior of the reduced average magnetization per grain, $\mathrm{m} / \mathrm{m}_{\mathrm{s}}$, where $m_{s}$ is the saturation value of the sample.

We begin our study by considering a sample with the same number of grains, average magnetic moment and average anisotropy constant of sample 1 , as defined in Table II, but with arbitrarily chosen size distributions.

First we assign a constant value of magnetization, $m_{i}=\bar{m}=869 \mu_{B}$, and uniform anisotropy constant, $K_{i}=\bar{K}=$ $1.58 \times 10^{6} \mathrm{erg} / \mathrm{cm}^{3}$, to all grains and anisotropy axis sorted from a uniform distribution. These choices represent a system in which all grains have the same size and shape, with anisotropy axis determined by the random alignment of the crystalline axis and the magnetic field. Figure 1 shows the results for this system. We notice that $T_{i r r}=T_{M}=8 \mathrm{~K}$ and that the peak of the ZFC is sharp.

Next we improve the description by considering a system with magnetizations sorted from a Gaussian distribution. In order to have a distribution quantitatively similar to the simulated one, we have chosen it to be centered at $\bar{m}=869 \mu_{B}$ with variance corresponding to the width of the distribution of sample 1 , that is, $\sigma=550 \mu_{B}$. We still keep the constant value of anisotropy $K$, i.e. all particles with the same shape, and anisotropy axis sorted from the uniform distribution. With these choices we have a system with particles of different sizes, but uniform shape, and with easy magnetization axis determined by the grain crystalline axis, as before. As can be seen in Figure 5 , the existence of particles with different sizes is not a sufficient condition for having $T_{i r r} \neq T_{M}$ as observed in experimental curves. A symmetrical distribution of sizes leads to curves similar to the ones obtained from a system of particles with uniform size in the sense that $T_{i r r}=T_{M}$ in both cases. However, because of the inclusion of a size distribution, we have now grains larger than in the previous case, and this is reflected in the larger values of those temperatures. Bigger particles with higher magnetizations weight more in the ZFC curve than the smaller ones, and because of its bigger size they yield bigger values of $T_{i r r}$. Therefore, the position of $T_{i r r}$ and $T_{M}$ shift to higher temperatures and now $T_{i r r}=T_{M}=12 \mathrm{~K}$. 


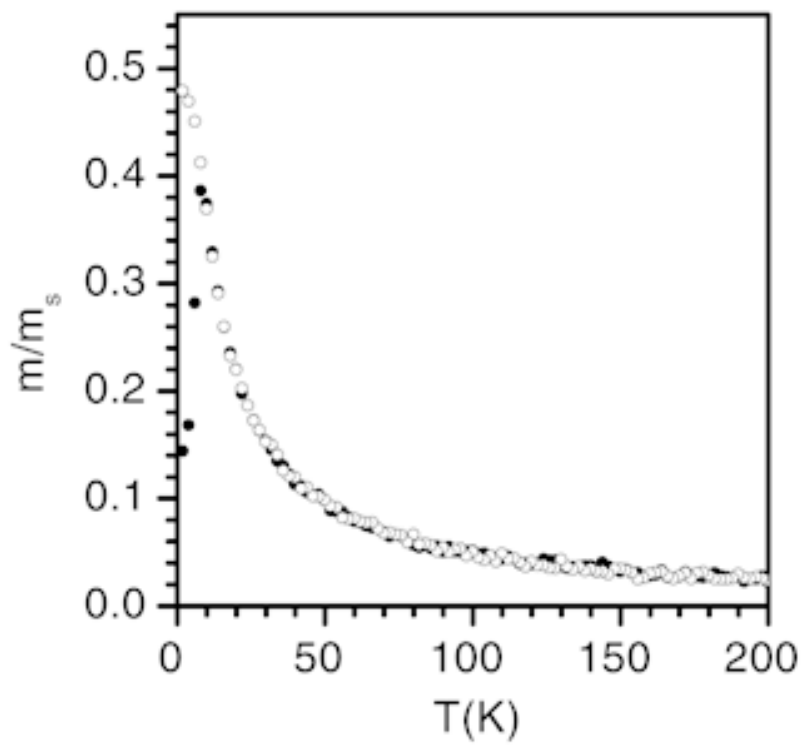

FIG. 4: Simulated ZFC-FC curves for a sample of 539 grains with constant magnetic moment $\left(=869 \mu_{B}\right)$ and anisotropy constant $\left(K=1.58 \mathrm{erg} / \mathrm{cm}^{3}\right)$ and anisotropy axis sorted from a uniform distribution. Since all particles have the same size, $T_{i r r}=T_{M}(=8 \mathrm{~K}$ in this case $)$, as expected.

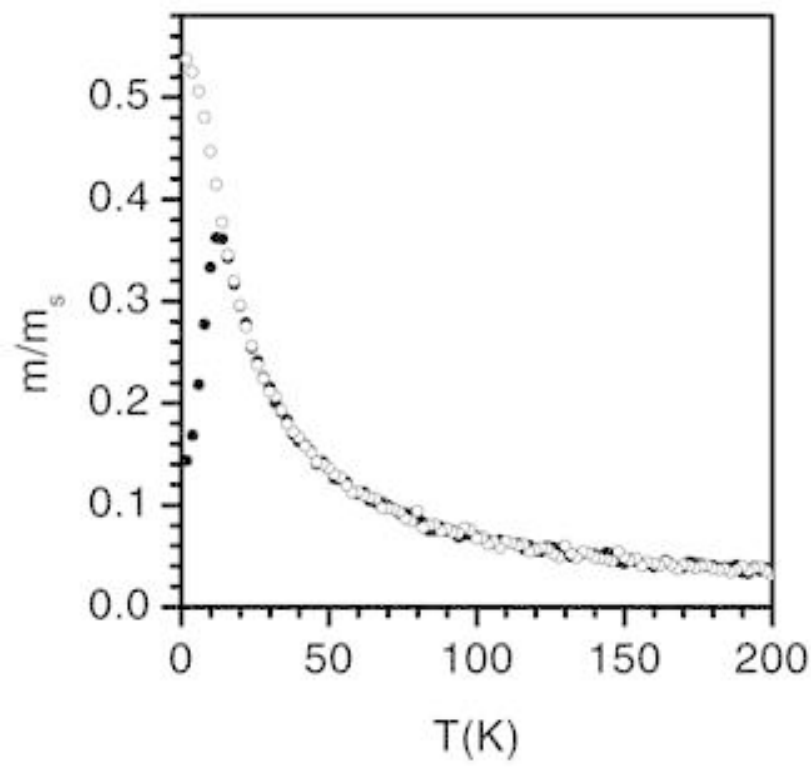

FIG. 5: Simulated ZFC-FC curves for a sample of 539 grains with Gaussian distribution of magnetic moments $\left(\bar{m}=869 \mu_{B}\right.$ and $\left.\sigma=550 \mu_{B}\right)$. All grains have the same anisotropy constant $\left(K=1.58 \mathrm{erg} / \mathrm{cm}^{3}\right)$ and the anisotropy axis are sorted from a uniform distribution. Although grains of different sizes are present, we still have $T_{i r r}=T_{M}$, indicating that the existence of a size distribution may not be the sole reason for having $T_{i r r}>T_{M}$. Here $T_{i r r}=T_{M}=12 \mathrm{~K}$.

The effect of the distribution profile can be clearly seen if we use the asymmetrical distribution generated by the CDS simulation, together with constant $K$ and uniformly distributed anisotropy axis. Figure 6 shows the resulting curves for this case, where $T_{i r r}>T_{M}\left(T_{i r r}=18 \mathrm{~K}\right.$ and $\left.T_{M}=13 \mathrm{~K}\right)$ and both are larger than the values obtained in the previous case, with the symmetrical distribution. Moreover the maximum value of $\mathrm{m} / \mathrm{m}_{\mathrm{s}}$ in the ZFC curve is lower as compared to the symmetrical distribution curve. In this case, the number of smaller particles is bigger, as compared to the monodisperse case. Some small particles are becoming disordered at the temperature of the 


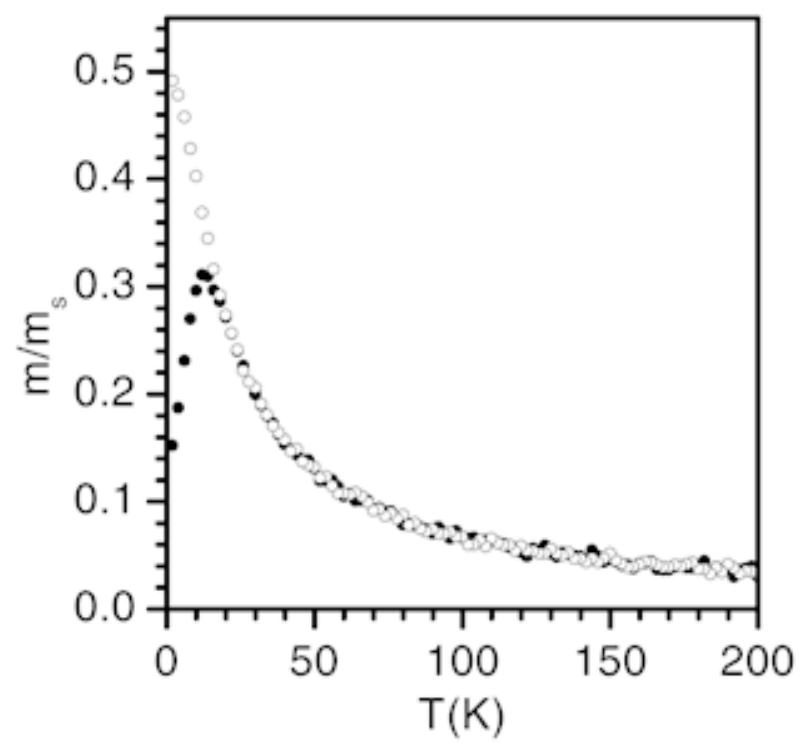

FIG. 6: Simulated ZFC-FC curves for a sample of 539 grains with a realistic distribution of magnetic moments from the CDS simulation in which $\bar{m}=869 \mu_{B}$. As in Fig. All grains have the same anisotropy constant $\left(=1.58 \mathrm{erg} / \mathrm{cm}^{3}\right)$ and the anisotropy axis are sorted from a uniform distribution. In this case $T_{i r r}=18 \mathrm{~K}$ and $T_{M}=13 \mathrm{~K}$.

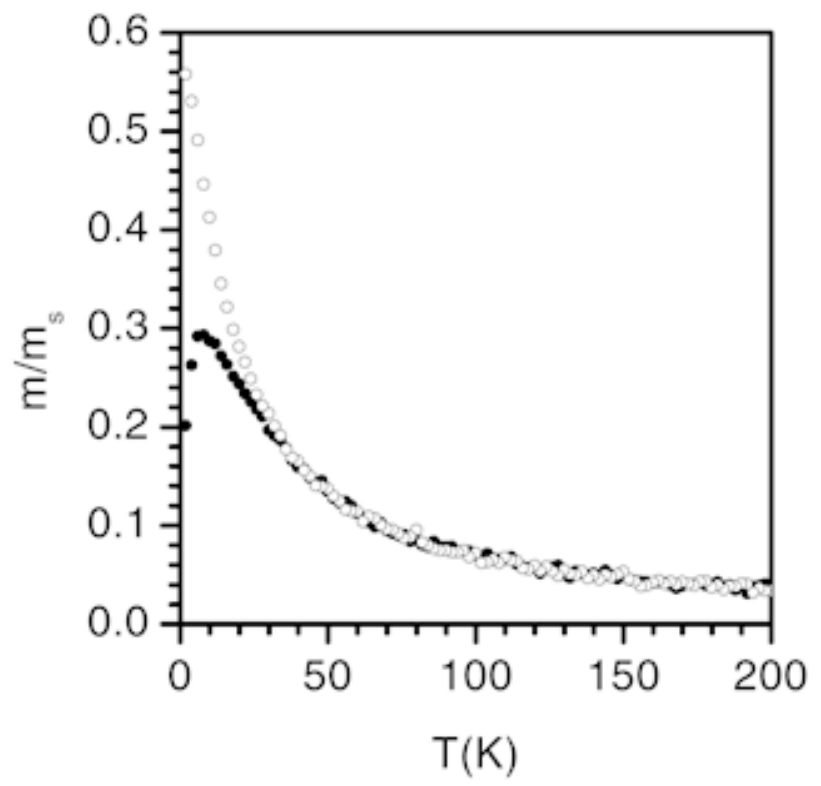

FIG. 7: Simulated ZFC-FC curves for sample 1 of Table III. The introduction of a realistic distribution of anisotropy constants generates a ZFC curve more similar to an experimental one (see Fig. 8), as the ZFC and FC curves separate at a temperature well below $T_{M}$. Here $T_{i r r}=36 \mathrm{~K} T_{M}=8 \mathrm{~K}$.

maximum magnetization of the sample, therefore, the magnetization peak in the ZFC curve is lower. Also for this reason, $T_{i r r}$ is larger.

Finally we use the complete information from the CDS simulation, that is, distribution of sizes, axis and anisotropy constants calculated from the simulated grains. In this case, $T_{i r r}=36 \mathrm{~K}$ and $T_{M}=8 \mathrm{~K}$.

From now on, all the simulated curves use the structural properties of the simulated alloy, as explained in Section IIB. 

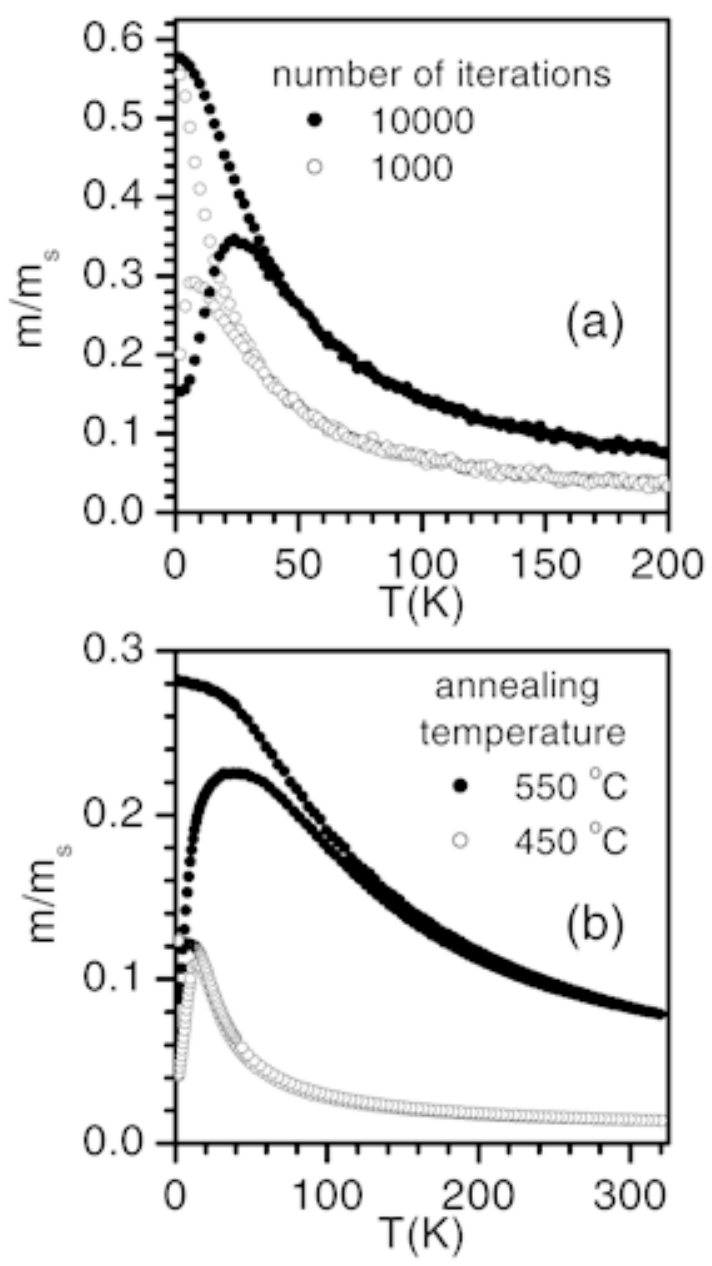

FIG. 8: (a) Simulated ZFC-FC curves for samples 1 and 2. (b) Experimental curves for a sample annealed for an hour at $450{ }^{\circ} \mathrm{C}$ and $550{ }^{\circ} \mathrm{C}$. Higher annealing temperatures facilitate the process of segregation, so we have a correspondence to a simulated sample with a larger number of iterations of the time evolution equation of the segregation process, Eq. (7).

\section{B. Effect of annealing}

Even for non interacting grains, annealing is capable of drastically changing the ZFC-FC curves. As the sample is brought to a temperature closer to the phase separation critical temperature, diffusion is enhanced and fabrication defects are relaxed. The overall effect is an isotropic sample with larger and fewer grains. For this reason we have chosen an annealed sample as our starting reference system. As the thermal treatment is repeated, grains grow larger and coalescence further decreases the number of grains. It is possible to achieve the same result in the simulated alloy simply by iterating more times the time evolution rule defined by equation (7). For this reason we assume that sample 2, collected 9000 iterations after sample 1 was collected, represents an annealed sample, as compared to sample 1. Using the same conversion factors defined for sample $1 \mathrm{in} \mathrm{s}$ ample 2, we guarantee consistency in the values of $m_{i}$ and $K_{i}$.

As expected for a sample with larger grains, both $T_{M}$ and $T_{i r r}$ are larger, and the maximum of both curves are higher. While a direct comparison is difficult, we can see that there is a qualitative agreement with experimental results, as illustrated in Fig. 8.(b). Because of the existence of bigger grains in sample 2, the mean value of the magnetization increases; therefore $T_{i r r}$ increases and also the low temperature limit of $\mathrm{m} / \mathrm{m}_{\mathrm{s}}$ on the FC curve. 


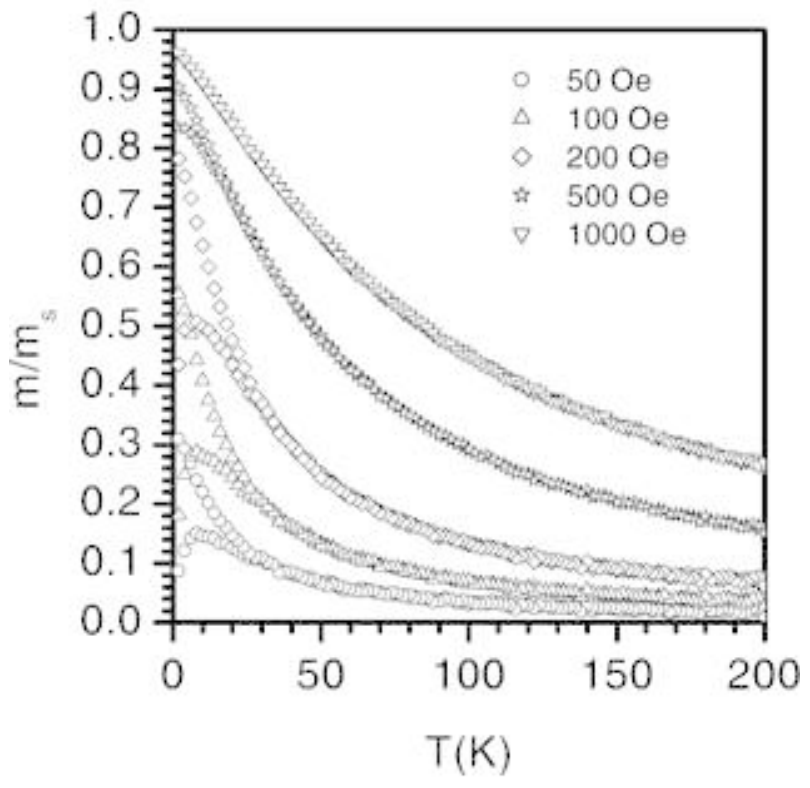

FIG. 9: Simulated ZFC-FC curves for sample 1 with different values of the applied field. Both $T_{i r r}$ and $T_{M}$ decrease with increasing field as can be seen in Table III.

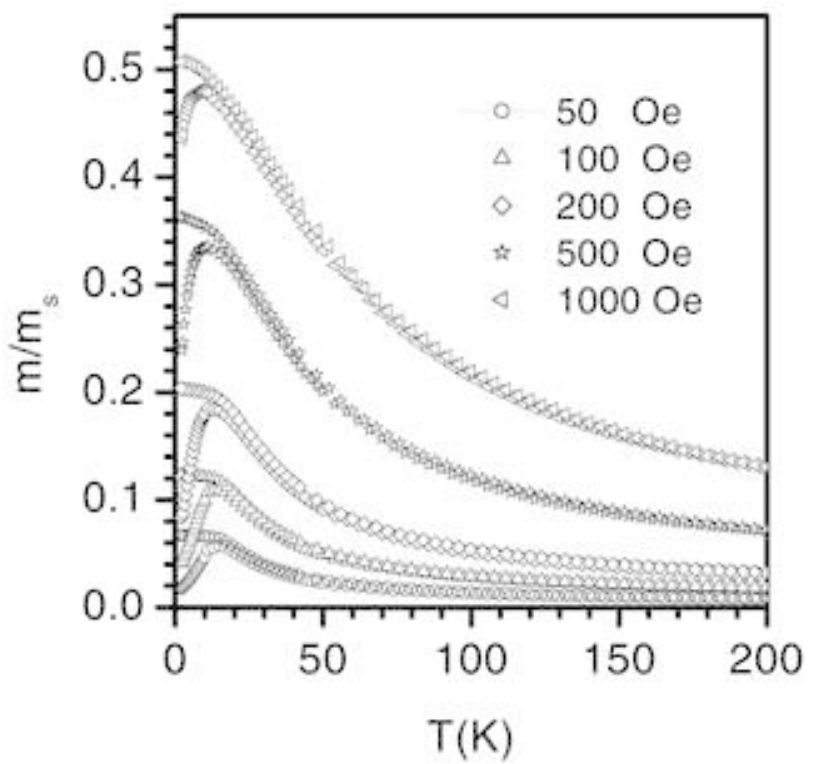

FIG. 10: Experimental ZFC-FC curves for a sample annealed at $450{ }^{\circ} \mathrm{C}$. Corresponding values of $T_{i r r}$ and $T_{M}$ are listed in Table 9 .

\section{Effect of external field}

Here we consider the effect of varying the magnitude of the applied magnetic field on sample 1. Figure 9 illustrates our results for fields in the range 50-1000 Oe. Simulated curves have the same qualitative behavior found in experiments, as depicted in Figure 10. That is, both ZFC and FC curves are dislocated to larger values of $\mathrm{m} / \mathrm{m}_{\mathrm{s}}$ as the field increases, and $T_{M}$ and $T_{i r r}$ decrease. Table III shows an estimation of the values of $T_{i r r}$ and $T_{M}$ for the simulated curves. 


\begin{tabular}{|c|c|c|c|c|}
\hline & \multicolumn{2}{|c|}{ Simulation } & \multicolumn{2}{c|}{ Experiment } \\
\hline field (Oe) & $T_{i r r}(K)$ & $T_{M}(\mathrm{~K})$ & $T_{i r r}(\mathrm{~K})$ & $T_{M}$ \\
\hline \hline 50 & 36 & 8 & 39 & 16 \\
\hline 100 & 36 & 8 & 38 & 15 \\
\hline 200 & 32 & 8 & 35 & 14 \\
\hline 500 & 26 & 4 & 22 & 11 \\
\hline 1000 & 14 & 2 & 19 & 9 \\
\hline
\end{tabular}

TABLE III: Values of $T_{i r r}$ and $T_{M}$ for the curves in Fig. 9 and 10.

Both values of temperature reflect the competition between thermal excitation and the energy barrier between the two axial orientations. $T_{i r r}$ is strongly influenced by the external field. As $T_{i r r}$ is related with equilibrium, i.e, it is the temperature above which the system behaves like a superparamagnet, by increasing the external field, the relaxation time diminishes. For superparamagnetic systems, the relaxation time at temperature $T$ is given by $\tau=\tau_{0} \exp \left(\frac{\Delta E}{k_{B} T}\right)$, where $\tau_{0}$ is a constant of the order of $10^{-9} \mathrm{~s}$ and $\Delta E$ is the energy barrier that each particle has to overcome to minimize its energy. Because the energy barrier decreases under an external magnetic field, so do $T_{M}$ and $T_{i r r}$, and the equilibrium state can be reached at lower temperatures.

\section{CONCLUSIONS}

There are several difficulties involved in the direct comparison of simulated and experimental curves, mostly because we are dealing with nonequilibrium states. The first issue is how to identify a simulated $\mathrm{Co}_{x} \mathrm{Cu}_{1-x}$ sample with a real one. As explained above, the CDS simulation deals with densities, and the space-time scale is undefined. As a zero order approximation we can force the simulated alloy to have the same average value of magnetic moment, but the variance of the grain size distribution in the simulation cannot be adjusted to be exactly the same as in the real system. Besides all this, there is also the problem of relating Monte Carlo steps with cooling and heating rates of the ZFC and FC curves, as discussed above. In summary, even though we have used experimental parameters to keep our simulated curves as close as possible to the real ones, a direct comparison of them is too ambitious. However, qualitative comparisons to determine the effect of each of the parameters of our model are valid, and the importance of relating anisotropy, size and shape distributions is stated.

The existence of a temperature $T_{i r r} \neq T_{M}$ marking the irreversible portion of the ZFC curve is already well understood to be a consequence of a grain size distribution 30 . What we observed in this work is that the sole existence of size distribution is not sufficient to produce a simulated ZFC curve similar to the experimental one. For symmetrical size distribution of the magnetic particles, $T_{M}$ coincide with $T_{i r r}$, within numerical accuracy as can be seen in Fig. 5. However, for non symmetrical particle size distributions, $T_{i r r}>T_{M}$ as shown in Fig. 6. The introduction of a distribution of anisotropy constants is a delicate point. As can be seen in Fig. 3, shape and size are correlated, so one cannot independently sort the values of magnetic moments and anisotropy constants, even when using the correct distributions. This effect has been so far neglected in simulations of granular systems. The qualitative comparison of the ZFC-FC curves corresponding to samples that have evolved in time with experimental curves for thermally treated samples reinforces this idea. As the binary alloy relaxes towards its equilibrium configuration, fewer and larger grains are present, and only the larger ones show appreciable anisotropy as shown in the scatter plot of anisotropy as a function of particle size for samples obtained after times (Fig. 3) for samples 1 and 2 obtained after different number of iterations.

\section{Acknowledgments}

A bilateral project Vitae/Fundación Andes is acknowledged by the authors. In Chile the groups received financial support from FONDECYT under grants \# 1010127 and 1990812, and Millennium Science Nucleus "Condensed Matter Physics" P99-135F. In Brazil, the authors acknowledge the support from FAPESP, FAPERJ, CAPES and CNPq.

${ }^{1}$ S. Y. C. M. S. Wei, P. R. Krauss, and P. B. Fischer, J. Appl. Phys. 76, 6673 (1994). 
${ }^{2}$ M. El-Hilo, K. O'Grady, and R. W. Chantrell, J. Appl. Phys. 76, 6811 (1994).

3 J. I. Gittleman, Y. Goldstein, and S. Bozowski, Phys. Rev. B 5, 3609 (1972).

${ }^{4}$ E. F. Ferrari, F. C. S. da Silva, and M. Knobel, Phys. Rev. B 56, 6086 (1997).

${ }^{5}$ B. J. Hickey, M. A. Howson, S. O. Musa, and N. Wiser, Phys. Rev. B 51, 667 (1995).

${ }^{6}$ N. Wiser, J. Magn. Magn. Mater. 159, 119 (1996).

7 D. A. Dimitrov and G. M. Wysin, Phys. Rev. B 54, 9237 (1996).

${ }^{8}$ P. Allia, M. Knobel, P. Tiberto, and F. Vinai, Phys. Rev. B 52, 15398 (1995).

${ }^{9}$ R. W. Chantrell, N. Walmsley, J. Gore, and M. Maylin, Phys. Rev. B 63, 024410 (2000).

${ }^{10}$ C. R. Pike, A. P. Roberts, and K. L. Verosub, J. Appl. Phys. 88, 967 (2000).

11 J. D. Gunton, M. S. Miguel, and P. S. Sahini (Academic Press, New York, 1983), vol. 8, chap. 2, p. 269.

12 S. M. Allen and J. W. Cahn, Acta Metall. 27, 1085 (1979).

13 K. Binder, Z. Pysik. 267, 313 (1974).

${ }_{14}^{14}$ F. C. S. da Silva, E. F. Ferrari, and M. Knobel, J. Appl. Phys. 86, 7170 (1999).

${ }^{15}$ Y. Oono and S. Puri, Phys. Rev. Lett. 58, 836 (1987).

${ }_{16}$ Y. Oono and S. Puri, Phys. Rev. A 38, 434 (1988).

17 Y. Oono and C. Yeung, J. Stat. Phys. 48, 593 (1987).

18 A. Shinozaki and Y. Oono, Phys. Rev. Lett. 66, 173 (1991).

19 Y. Oono and M. Bahiana, Phys. Rev. Lett. 61, 1109 (1988).

20 M. Mondello and N. Goldenfeld, Phys. Rev. A 42, 5865 (1990).

${ }^{21}$ M. S. O. Massunaga, M. Paniconi, and Y. Oono, Phys. Rev. E 56, 723 (1997).

${ }^{22}$ S. Martins, W. Morgado, M. Massunaga, and M. Bahiana, Phys. Rev. E 61, 4118 (2000).

${ }^{23}$ S. Puri and Y. Oono, Phys. Rev. A 38, 1542 (1988).

${ }^{24}$ J. Hoshen and R. Kopelman, Phys. Rev. B 14, 3438 (1976).

${ }^{25}$ R. H. Yu, J. Zhu, X. Zhang, and M. Knobel, Materials Science and Technology 12, 464 (1996).

${ }^{26}$ B. D. Cullity, Introduction to magnetic materials (Addison-Wesley, 1972).

${ }_{27}^{27}$ R. H. Yu, X. X. Zhang, J. Tejada, J. Zhu, M. Kobel, P. Tiberto, P. Allia, and F. Vinai, J. Appl. Phys. 78, 5062 (1995).

${ }_{28}$ G. Bertotti, Hysteresis in magnetism. For Physicists, materials scientists, and engineers (Academic Press, 1998).

${ }^{29}$ D. Altbir, P. Vargas, and J. d' Albuquerque e Castro, Phys. Rev. B 54, R6823 (1996).

30 J. L. Dormann, D. Fiorani, and E. Tronc, Adv. Chem. Phys. XCVIII, 283 (1997). 